\title{
Fatty acid profile of fish following a change in dietary fatty acid source: model of fatty acid composition with a dilution hypothesis
}

\author{
J. H. Robin ${ }^{a^{*}}$, C. Regost ${ }^{a}$, J. Arzel ${ }^{a}$ and S. J. Kaushik ${ }^{b}$ \\ ${ }^{a}$ Fish Nutrition Laboratory INRA-IFREMER, IFREMER Centre de Brest, 29280 Plouzané, France \\ ${ }^{\mathrm{b}}$ Fish Nutrition Laboratory INRA-IFREMER, INRA, 64310 Saint-Pée-sur-Nivelle, France \\ * : Corresponding author : jrobin@ifremer.fr
}

\begin{abstract}
The fatty acid (FA) content of fish is generally said to reflect fatty acid composition of the diet. In fact, incorporation of FA into tissues is modulated by various metabolic factors, and final composition will depend upon the initial FA content, cumulative intake of dietary fatty acids, growth rate and duration. Analysis of time course of changes in FA composition should be easier with animals having different initial FA profiles, which are subsequently fed a diet with the same FA composition.

Data from two studies, one with brown trout and another with turbot were used. Fish were first fed with diets containing one of three different oils (soybean oil (SO), linseed oil (LO) and fish oil (FO)), and subsequently fed the same fish oil-based diet (washout period). If we suppose a model fish having the same initial composition as those fed vegetable oil and which incorporate fatty acid in the same way as the control fish always fed fish oil, we may compute a model of dilution of initial fatty acid content with increasing growth and absolute fat deposition. Experimental data can be compared with a reference fatty acid profile given by this model for the same fatty acid increase.

Application of the model to experimental data shows that while muscle neutral lipid (NL) FAs roughly follow this dilution model, those of muscle polar lipids $(\mathrm{PL})$ undergo much faster changes than model values based on increase of total polar lipid quantities. Among observed differences between the model and experimental values, DHA is of particular interest as this fatty acid displays lower change rates (significant in turbot neutral lipids) than expected in contrast to other fatty acids.
\end{abstract}

Keywords: Fatty acids; Fish; Model; Dietary change 


\section{Introduction}

Fatty acid composition of fillets reflects the fatty acid composition of diets, although the differences in fatty acid composition among groups of fish are less than the differences in fatty acid composition among their respective diets (Hardy et al., 1987). In fact, incorporation of FA into tissues is under various metabolic influences such as preferential incorporation (Linares and Henderson, 1991), $\beta$-oxidation (Kiessling and Kiessling, 1993), lipogenic activity, or fatty acid elongation and desaturation processes (Henderson and Sargent, 1985). It is thus difficult to predict fish fatty acid composition from dietary components. The fatty acid composition may also be affected environmental factors (Tocher and Sargent, 1990) or size or age of animals (Kiessling et al., 2001), affecting metabolic activity.

It is also difficult to predict the time required to reach a relatively stable FA composition in fish fed a particular diet. It could be relatively short (Skonberg et al., 1994), or continue to evolve over a longer period (Tidwell and Robinette, 1990). With fast growing young fish, it is possible to obtain a rather complete effect of dietary FA on fish FA composition in a relatively short period of time. In the case of large fish, since the relative weight increment is small, the initial FA composition will continue to have a strong influence on final composition. It is thus essential that following a change in dietary FA source, FA composition must be interpreted taking into account the initial FA content, intake, increase in tissue mass and duration.

The use of vegetable oils in fish diets has an influence on flesh quality (; Thomassen and Røsjø, 1989; Sérot et al., 2001), at least in their fatty acid profile. Considering the interest in fish n-3 fatty acids for human health (Nettleton, 1991), fatty acid profile of fish fed plant oils might be considered as having possible negative effects in this regard. After using diets containing vegetable oils, a return to a fish oil diet is one way of reducing this adverse effect (Regost et al., 2003). This "washout" period is supposed to return fatty acid composition or 
quality parameters back to values similar to those of fish continuously fed a fish oil diet. However, Regost (2001) found that after two months on the same diet the fatty acid profiles of fish initially fed on different oil sources still differed among experimental groups, even if the initial differences were partly reduced. In order to clearly analyse the rate of change in fatty acid composition of the whole animal or a particular tissue, we need a working model.

A simple dilution model considers that dietary fatty acids are incorporated as such into tissues without any mobilisation or turnover of pre-existing ones. Another assumption is that the same species of fish under similar culture conditions, would incorporate dietary FA in the same manner, irrespective of the initial fatty acid composition. The aim of the present study was to propose and evaluate such a theoretical model based on dilution of initial fatty acids by those incorporated from a diet, as a tool of comparison.

\section{Materials and methods}

Data from two studies (Regost, 2001), one with brown trout (Salmo trutta) and another with turbot (Psetta maxima), with fish close to market size, were used as examples. These studies involved feeding fish to satiation over 3 months with fish meal based diets containing, respectively, 15 and 9\% added: fish oil (FO), soybean oil (SO) or linseed oil (LO), and subsequently fed during 2 months on the same FO based diet (washout period). Details of protocols and analysis can be found in Regost (2001) and Regost et al. (2003). For lipid analyses, extraction of muscle lipids was done according to Folch et al. (1957), with chloroform being replaced by dichloromethane. The separation of neutral lipids (NL) and Polar lipids (PL) was performed according to Juaneda and Rocquelin (1985), on silica cartridges (Sep-Pack, Waters). Fatty acids methyl esters (FAME) were prepared following the method of Morrison and Smith (1964) with a known quantity of heneicosanoic acid as internal standard. FAME were separated by gas chromatography (Auto-system Perkin-Elmer 
with a flame ionisation detector, BPX 70 capillary column: $25 \mathrm{~m}$ x $0.22 \mathrm{~mm}$ i.d. x $0.25 \mu \mathrm{m}$ d.f.; split-splitless injector, with helium as carrier gas). The individual fatty acid methyl esters were identified by comparing the retention times of authentic standard mixtures, and quantified relatively to the internal standard.

If we suppose a model fish having the same initial composition as those fed plant oil and which incorporate fatty acids in the same way as the control fish always fed fish oil, we may compute a model of dilution of initial fatty acid content with increasing growth and absolute fat deposition. The dilution of an initial quantity of a mixture of fatty acids by an increasing quantity of a new mixture can be logically described by the following equation for each fatty acid:

$$
P i(t)=P i f+\frac{P i o-P i f}{Q(t) / Q o}
$$

Where $P i$ is the percentage of the i fatty acid, with $o$ for its original (initial) value, and $f$ for its final value. Qo represents the initial quantity of total fatty acids and $Q(t)$ the same at time $t$. The same $Q(t) / Q o$ serves for calculation of all fatty acids in the same tissue profile. This formula can be used to describe the expected change in fatty acids either in a fish or in a tissue or in a fraction (polar or neutral lipids). This model represents an expected minimal change if there is no mobilization or turnover of FA, and can be compared with experimental values.

Here the values of the fatty acid composition of neutral and polar fractions of lipids in the muscle of brown trout and turbot were used from the study of Regost (2001). Pio is the percentage of each fatty acid in a fatty acid profile (as a fraction of the muscle) of animals previously fed SO and LO diets at the beginning of the washout period. Pif represents that of the reference group always fed on fish oil, at the end of the experiment. 
The evaluation of quantities $Q o$ and $Q(t)$ should take into account at each time $o$ and $t$ the weight, proportion of studied tissue versus animal weight, and lipid quantity of the fraction of the tissue, and FA profile in the lipid. However, as $Q(t) / Q o$ is a ratio, calculation can be simplified when some proportions can be considered as constant over time. On the basis of results obtained, muscle weight to body weight, level of polar lipids in the muscle, and percent total fatty acid in lipid fractions were considered as constant. Then evaluations of $Q(t) / Q o$ for muscle neutral lipid took into consideration variation of neutral lipid content and growth of whole fish, while weight increase was the only factor increasing the quantity of polar lipids. Mean values were used for each treatment of 6 replicate fish.

The hypothesis that the value calculated by the dilution equation for each fatty acid could represent an expected mean of the experimental data was checked by a test of conformity (Dagnelie, 1969).

For comparison of whole fatty acid profiles, we used the coefficient of distance $D$ (McIntire et al., 1969):

$$
D(h-j)=\left(\sum_{i=1}^{n}(P i h-P i j)^{2}\right)^{1 / 2}
$$

(equation ii)

Where the $D(h-j)$ is distance between samples $h$ and $j$, and Pih and Pij are percentage of the fatty acid $i$ in samples $h$ and $j$, for each $i$ fatty acid.

This $D$ value has been used in ecological studies focusing on the relationship between fatty acid profiles in natural communities. Bottino (1974) found a mean $D$ value of 6 to 7.7 for analysis of same species of animal in natural environment, and a $D$ value around 2 between replicates of the same sample. 


\section{Results}

During the washout period, mean fish weight increase from 2.4 to $3.0 \mathrm{~kg}$ for brown trout and from 0.85 to $1.16 \mathrm{~kg}$ for turbot without differences between treatments, there was an increase in muscle fat content of fish of both treatments and species. These increases affected neutral lipids (NL) only while polar lipid (PL) content remained constant on a weight basis. Then a higher relatively fatty acid increase per animal $(Q(t) / Q o)$ occurred in muscle neutral lipids (1.75 and 1.74 for turbot previously fed LO and SO diets, and 1.25 and 1.89 for brown trout fed SO and LO diets) than in muscle polar lipids (1.42 and 1.38 for turbot previously fed LO and SO diets, and 1.18 and 1.23 for brown trout fed SO and LO diets). Using these coefficients with initial and control fish fatty acid profiles, values corresponding to the equation (i) were calculated for each species and treatment. Values obtained were compared to observed values (Tables 1 and 2). In the muscle of fish previously fed vegetable oils, percentage of each fatty acid after two months of washout period $(\operatorname{Pi}(t))$ showed an intermediate value between the initial value (Pio) and the corresponding value of the FO group (Pif). The fatty acids present in high amounts in the plant oils (18:2n-6 and 18:3n-3) decreased while those more characteristic of fish oil (22:6n-3, 20:5n-3 or n-11 monenes) increased, except 22:6n-3 in polar lipids of turbot and LO-fed brown trout. Then for each fatty acid, the evolution must be followed considering Pio and Pif values. In tables 1 and 2, the "_." sign following each comparison indicates that the observed percentage corresponds to a lower change than expected by the dilution model. Over 134 comparisons, this occurred in 33 cases, only 7 of which corresponded to significant differences between observed and calculated values. In contrast, 67 significant differences were found between observed and calculated values with a change rate higher than that expected by dilution, most of them occurred in polar lipids . 
In turbot (Table 1), mean experimental data were relatively close to values given by the model, although significant differences were observed in most cases. Among those fatty acids showing such differences, the experimental data were closer to its corresponding Pif than described by the model, showing that the rate of change was clearly higher than predicted by the model. The exceptions encountered were DHA in neutral lipids (LN) and 22:5n-3 in NL of LO group which on the contrary showed significantly smaller changes than predicted by the model.

In brown trout muscle (Table 2), PL fatty acid profiles of fish previously fed vegetable oils were relatively similar to that of the FO group, more closely than that expected by dilution. In neutral lipids, less significant differences were observed between experimental data and model values than in PL. In NL of the SO group, experimental data exhibited a higher rate of change than the model while the reverse was observed in the LO group. However only a few fatty acids (20:1n-9; 18:1n-11, 20:1n-11, 18:3n-3) differed significantly from the model in this latter group.

An overall evaluation of the changes in fatty acid profiles compared to the dilution model can be observed using " $D$ " values of distances (Table 3). Distances between observed and model fatty acid spectra ranged from 1.7 to 3.3 in muscle NL, and from 2.6 to 3.9 in PL for both species. For each species and treatment, the distances between the model and observations were lower in NL than in PL, despite higher initial distances between FO and vegetable groups in neutral lipids than in polar lipids. Except for the LO group of brown trout, the distance between observed profiles and that of the FO group was lower than $D$ values between the model and the FO group. The calculated distance coefficient provides another way to estimate the evolution of fatty acid profiles, by computing the relative decrease of distance between treatments during the washout period (Table 3). This index did not show such differing rates of change between neutral and polar lipids. 
Table 1. Comparison of calculated $(P i(t)$ cal) and observed $(P i(t)$ obs \pm se) percent of total fatty acids in muscular neutral and polar lipids of turbot after 2 months of washout on fish oil diet. Some minor fatty acids were omitted.

\begin{tabular}{|c|c|c|c|c|c|c|c|c|c|c|c|}
\hline \multirow{2}{*}{$\underline{\text { previous diet }}$} & \multicolumn{2}{|l|}{$\mathrm{FO}$} & \multicolumn{5}{|l|}{ so } & \multicolumn{4}{|l|}{ LO } \\
\hline & Pif & Pio & Pi(t)obs. & $P i(t) \mathrm{cal}$ & & & io & Pi(t)obs. & $P i(t)$ cal & & \\
\hline \multicolumn{12}{|l|}{ Neutral lipids } \\
\hline $14: 0$ & 6.9 & 3.3 & $5.5 \pm 0.2$ & 4.9 & $\star \star$ & & 3.2 & $5.2 \pm 0.1$ & 4.8 & * & \\
\hline $16: 0$ & 14.4 & 12.9 & $14.1 \pm 0.2$ & 13.5 & * & & 11.1 & $13.1 \pm 0.2$ & 12.5 & * & \\
\hline 18:0 & 1.5 & 2.5 & $1.8 \pm 0.0$ & 2.1 & ** & & 2.7 & $1.8 \pm 0.1$ & 2.2 & $\star *$ & \\
\hline $16: 1 n-7$ & 5.9 & 3.7 & $5.1 \pm 0.2$ & 4.6 & & & 3.5 & $4.8 \pm 0.1$ & 4.5 & & \\
\hline $18: 1 n-7$ & 2.6 & 2.4 & $2.5 \pm 0.0$ & 2.5 & & & 2.1 & $2.3 \pm 0.0$ & 2.3 & & - \\
\hline $18: 1 n-9$ & 14.4 & 17.2 & $15.8 \pm 0.1$ & 16.0 & * & & 15.6 & $15.3 \pm 0.2$ & 15.1 & & - \\
\hline $20: 1 n-9$ & 9.2 & 4.2 & $7.1 \pm 0.1$ & 6.3 & ** & & 3.8 & $6.8 \pm 0.2$ & 6.1 & * & \\
\hline $22: 1 n-9$ & 1.1 & 0.7 & $0.9 \pm 0.0$ & 0.9 & * & & 0.7 & $0.9 \pm 0.0$ & 0.9 & ** & \\
\hline $20: 1 n-11$ & 1.2 & 0.6 & $0.9 \pm 0.0$ & 0.8 & $* *$ & & 0.5 & $0.9 \pm 0.0$ & 0.8 & & \\
\hline $22: 1 n-11$ & 11.3 & 4.5 & $8.7 \pm 0.2$ & 7.4 & ** & & 4.0 & $8.3 \pm 0.2$ & 7.1 & $* *$ & \\
\hline $18: 2 n-6$ & 5.3 & 24.2 & $14.1 \pm 0.6$ & 16.1 & * & & 12.1 & $8.6 \pm 0.1$ & 9.2 & ** & \\
\hline $20: 2 n-6$ & 0.5 & 1.4 & $0.9 \pm 0.0$ & 1.0 & & & 0.7 & $0.6 \pm 0.0$ & 0.6 & & \\
\hline $20: 4 n-6$ & 0.4 & 0.5 & $0.4 \pm 0.0$ & 0.4 & $\star *$ & & 0.5 & $0.4 \pm 0.0$ & 0.5 & * & \\
\hline $18: 3 n-3$ & 1.2 & 3.0 & $2.0 \pm 0.1$ & 2.2 & * & & 18.3 & $10.4 \pm 0.6$ & 11.0 & & \\
\hline $18: 4 n-3$ & 2.2 & 1.2 & $1.7 \pm 0.1$ & 1.6 & & & 1.1 & $1.6 \pm 0.1$ & 1.6 & & \\
\hline $20: 5 n-3$ & 6.8 & 4.8 & $5.7 \pm 0.1$ & 5.6 & & & 4.5 & $5.5 \pm 0.1$ & 5.5 & & \\
\hline $22: 5 n-3$ & 1.8 & 1.5 & $1.5 \pm 0.0$ & 1.6 & & - & 1.5 & $1.5 \pm 0.0$ & 1.7 & $* *$ & - \\
\hline$\underline{22: 6 n-3}$ & 11.1 & 9.2 & $9.4 \pm 0.1$ & 10.0 & $\star *$ & - & 9.8 & $9.4 \pm 0.2$ & 10.4 & $\star *$ & - \\
\hline \multicolumn{11}{|l|}{ Polar lipids } & \\
\hline $14: 0$ & 1.9 & 0.8 & $1.6 \pm 0.1$ & 1.2 & $\star *$ & & 0.8 & $1.6 \pm 0.1$ & 1.1 & $\star *$ & \\
\hline $16: 0$ & 22.9 & 20.3 & $22.3 \pm 0.2$ & 21.1 & ** & & 19.5 & $21.5 \pm 0.5$ & 20.5 & & \\
\hline 18:0 & 5.6 & 6.2 & $5.9 \pm 0.2$ & 6.0 & & & 6.5 & $6.2 \pm 0.2$ & 6.3 & & \\
\hline $16: 1 n-7$ & 1.6 & 0.8 & $1.5 \pm 0.1$ & 1.0 & $* *$ & & 0.7 & $1.4 \pm 0.1$ & 0.9 & $* *$ & \\
\hline $18: 1 n-7$ & 2.0 & 1.7 & $2.0 \pm 0.1$ & 1.8 & * & & 1.6 & $2.0 \pm 0.1$ & 1.7 & * & \\
\hline $18: 1 n-9$ & 11.2 & 10.6 & $11.5 \pm 0.3$ & 10.8 & & & 10.7 & $11.9 \pm 0.3$ & 10.8 & * & \\
\hline $20: 1 n-9$ & 3.7 & 1.6 & $3.0 \pm 0.2$ & 2.2 & $* *$ & & 1.6 & $3.2 \pm 0.1$ & 2.1 & $* *$ & \\
\hline $18: 1 n-11$ & 1.1 & 0.3 & $0.7 \pm 0.3$ & 0.5 & & & 0.4 & $0.2 \pm 0.2$ & 0.6 & & - \\
\hline $22: 1 n-11$ & 1.3 & 0.5 & $1.2 \pm 0.1$ & 0.7 & ** & & 0.4 & $1.1 \pm 0.0$ & 0.7 & ** & \\
\hline $18: 2 n-6$ & 4.5 & 18.7 & $11.8 \pm 0.3$ & 14.5 & ** & & 10.7 & $7.4 \pm 0.2$ & 9.0 & ** & \\
\hline $20: 2 n-6$ & 0.4 & 1.2 & $0.7 \pm 0.0$ & 0.9 & $\star *$ & & 0.7 & $0.5 \pm 0.0$ & 0.6 & $* *$ & \\
\hline $20: 4 n-6$ & 2.4 & 2.0 & $2.1 \pm 0.1$ & 2.1 & & - & 2.0 & $2.2 \pm 0.1$ & 2.1 & & \\
\hline $18: 3 n-3$ & 0.4 & 1.1 & $0.7 \pm 0.0$ & 0.9 & ** & & 8.1 & $3.9 \pm 0.2$ & 6.0 & $\star *$ & \\
\hline $20: 5 n-3$ & 10.1 & 5.8 & $7.9 \pm 0.1$ & 7.1 & ** & & 6.1 & $8.2 \pm 0.2$ & 7.2 & ** & \\
\hline $22: 5 n-3$ & 2.4 & 1.9 & $2.0 \pm 0.1$ & 2.1 & & - & 2.0 & $2.0 \pm 0.1$ & 2.1 & & - \\
\hline $22: 6 n-3$ & 26.6 & 24.9 & $24.0 \pm 0.6$ & 25.4 & & - & 25.7 & $25.4 \pm 0.6$ & 26.0 & & - \\
\hline
\end{tabular}

Significant differences between calculated and observed percentages are indicated $* \mathrm{P}<0.05$ and $* * \mathrm{P}<0.01$. The "_." symbol indicates a less than expected evolution of the fatty acid compared to the model of dilution. 
Table 2 Comparison of calculated (Pi $(t)$ cal) and observed ( $P i(t)$ obs $\pm s e)$ percent of total fatty acids in muscular neutral and polar lipids of brown trout after 2 months of washout on fish oil diet. Some minor fatty acids were omitted.

\begin{tabular}{|c|c|c|c|c|c|c|c|c|c|c|}
\hline \multirow[t]{2}{*}{ previous diet } & \multicolumn{2}{|l|}{$\mathrm{FO}$} & \multicolumn{4}{|l|}{ SO } & \multicolumn{4}{|l|}{ LO } \\
\hline & Pif & Pio & Pi $(t)$ obs & $P i(t) \mathrm{cal}$ & & Pio & $P i(t)$ obs & $P i(t) \mathrm{cal}$ & & \\
\hline \multicolumn{10}{|l|}{ Neutral lipids } & \\
\hline 14:0 & 4.3 & 2.6 & $3.2 \pm 0.1$ & 2.9 & & 3.1 & $3.5 \pm 0.1$ & 3.7 & & - \\
\hline 16:0 & 16.4 & 14.5 & $14.3 \pm 0.3$ & 14.9 & - & 13.6 & $14.8 \pm 0.2$ & 14.9 & & - \\
\hline 18:0 & 3.1 & 3.4 & $3.1 \pm 0.1$ & 3.4 & * & 3.3 & $3.3 \pm 0.1$ & 3.2 & & - \\
\hline $16: 1 \mathrm{n}-7$ & 6.1 & 3.2 & $4.0 \pm 0.3$ & 3.8 & & 3.7 & $4.7 \pm 0.2$ & 4.8 & & - \\
\hline $18: 1 n-7$ & 3.3 & 2.2 & $2.6 \pm 0.1$ & 2.5 & & 2.3 & $2.7 \pm 0.1$ & 2.8 & & - \\
\hline $18: 1 n-9$ & 20.7 & 21.8 & $20.9 \pm 030$ & 21.6 & & 19.3 & $20.3 \pm 0.4$ & 20.0 & & \\
\hline 20:1n-9 & 5.2 & 3.2 & $4.2 \pm 0.2$ & 3.6 & * & 3.8 & $4.0 \pm 0.1$ & 4.5 & ** & - \\
\hline $18: 1 n-11$ & 1.2 & 0.7 & $0.9 \pm 0.0$ & 0.8 & & 0.8 & $0.9 \pm 0.0$ & 1.0 & ** & - \\
\hline $20: 1 n-11$ & 1.1 & 0.6 & $0.7 \pm 0.0$ & 0.7 & & 0.7 & $0.8 \pm 0.0$ & 0.9 & ** & - \\
\hline $22: 1 n-11$ & 4.8 & 2.7 & $3.8 \pm 0.2$ & 3.2 & * & 3.4 & $3.7 \pm 0.2$ & 4.1 & & - \\
\hline $18: 2 n-6$ & 7.1 & 24.8 & $18.8 \pm 1.3$ & 21.3 & & 13.0 & $10.7 \pm 040$ & 10.2 & & - \\
\hline $20: 2 n-6$ & 0.6 & 1.1 & $1.0 \pm 0.1$ & 1.0 & & 0.7 & $0.7 \pm 0.0$ & 0.7 & & \\
\hline $20: 4 n-6$ & 0.6 & 0.3 & $0.4 \pm 0.0$ & 0.4 & & 0.3 & $0.4 \pm 0.0$ & 0.4 & & - \\
\hline $18: 3 n-3$ & 1.4 & 5.7 & $4.1 \pm 0.3$ & 4.9 & * & 15.2 & $10.1 \pm 0.4$ & 8.7 & * & - \\
\hline $18: 4 n-3$ & 1.3 & 1.1 & $1.2 \pm 0.0$ & 1.1 & & 1.5 & $1.4 \pm 0.0$ & 1.4 & & - \\
\hline $20: 4 n-3$ & 1.4 & 0.9 & $1.1 \pm 0.0$ & 1.0 & ** & 1.6 & $1.5 \pm 0.0$ & 1.5 & & \\
\hline $20: 5 n-3$ & 4.5 & 2.0 & $3.0 \pm 0.2$ & 2.5 & * & 2.8 & $3.4 \pm 0.1$ & 3.6 & & - \\
\hline $22: 5 n-3$ & 2.2 & 0.9 & $1.5 \pm 0.1$ & 1.2 & ** & 1.2 & $1.6 \pm 0.1$ & 1.7 & & - \\
\hline $22: 6 n-3$ & 11.7 & 5.1 & $8.0 \pm 0.5$ & 6.4 & * & 6.7 & $8.4 \pm 0.2$ & 9.0 & & - \\
\hline \multicolumn{11}{|l|}{ Polar lipids } \\
\hline 14:0 & 1.6 & 0.9 & $1.3 \pm 0.1$ & 1.1 & * & 1.2 & $1.7 \pm 0.1$ & 1.3 & ** & \\
\hline 16:0 & 21.2 & 19.1 & $21.2 \pm 0.3$ & 19.5 & ** & 18.1 & $20.4 \pm 0.3$ & 18.7 & ** & \\
\hline 18:0 & 5.4 & 5.3 & $5.8 \pm 0.2$ & 5.4 & * & 5.4 & $5.4 \pm 0.1$ & 5.4 & & - \\
\hline $16: 1 \mathrm{n}-7$ & 1.3 & 0.8 & $0.9 \pm 0.1$ & 0.9 & & 0.9 & $1.3 \pm 0.1$ & 0.9 & & \\
\hline $18: 1 n-7$ & 1.9 & 1.3 & $1.7 \pm 0.1$ & 1.4 & * & 1.4 & $1.7 \pm 0.1$ & 1.5 & * & \\
\hline $18: 1 n-9$ & 5.6 & 6.7 & $5.9 \pm 0.3$ & 6.5 & * & 6.0 & $6.3 \pm 0.3$ & 5.9 & & - \\
\hline $18: 2 n-6$ & 1.7 & 7.5 & $4.2 \pm 0.2$ & 6.6 & ** & 3.5 & $2.7 \pm 0.1$ & 3.2 & ** & \\
\hline $20: 3 n-6$ & 0.3 & 1.9 & $0.9 \pm 0.0$ & 1.6 & ** & 0.6 & $0.4 \pm 0.0$ & 0.5 & ** & \\
\hline $20: 4 n-6$ & 1.5 & 1.7 & $1.6 \pm 0.0$ & 1.7 & * & 1.1 & $1.3 \pm 0.0$ & 1.2 & ** & \\
\hline $18: 3 n-3$ & 0.5 & 2.6 & $1.6 \pm 0.2$ & 2.3 & * & 6.0 & $3.7 \pm 0.2$ & 5.0 & ** & \\
\hline $20: 4 n-3$ & 1.0 & 1.1 & $1.0 \pm 0.0$ & 1.1 & & 1.6 & $1.3 \pm 0.0$ & 1.5 & * & \\
\hline $20: 5 n-3$ & 8.0 & 6.8 & $6.9 \pm 0.3$ & 7.0 & - & 7.3 & $7.4 \pm 0.1$ & 7.4 & & \\
\hline $22: 5 n-3$ & 2.5 & 2.0 & $2.1 \pm 0.1$ & 2.1 & & 2.0 & $2.3 \pm 0.1$ & 2.1 & * & \\
\hline $22: 6 n-3$ & 44.4 & 38.7 & $41.6 \pm 0.9$ & 39.6 & * & 41.3 & $40.8 \pm 0.8$ & 41.9 & & - \\
\hline
\end{tabular}

Same foot note as in Table 2. 
Table III: Distances between fatty acid spectra of observed and calculated after 2 months of washout, with control FO fed fish (FOfish) and with initial fatty acid spectra at the beginning of washout period, in Neutral lipids (NL) and Polar lipids (PL) of fish muscle.

\begin{tabular}{llll}
\hline & $\begin{array}{l}\text { Turbot } \\
\text { Previous diet }\end{array}$ & LO & \multicolumn{2}{c}{ Brown trout } \\
SO
\end{tabular}

Note: mean $D$ values found between replicates were 2.5 for turbot NL, 4.0 for brown trout NL and around 3 for PL of both species.

\section{Discussion}

The proposed model includes several approximations when applied to experimental data. The composition of FO fish was considered as the expected final composition of the other treatments, which is reasonable, although the FA composition of this FO group was not totally stabilised. The fatty acid profiles at the beginning and at the end of the washout period were compared by computing distances for each species. The average distances were 2.4 and 3.6 for NL and PL of turbot and 2.5 and 3.3 for both PL and NL of brown trout. These distances correspond to a normal variability within the same population, compared to distances within replicates (Bottino, 1974). In practice, the aim of the washout is only to obtain fish quality which could be considered as normal for a marine product. Thus, the small variation observed here is well within an intra-population tolerance limits.

The quantities $Q o$ and $Q(t)$ are more difficult to evaluate under practical conditions. This requires the compilation of various data (total weight, tissue to total weight ratio, lipid content, fatty acid content) each implying variability and analytical errors. The use of the ratio $Q(t) / Q o$ has the advantage of simplifying the calculation when some values can be considered as similar or subject to little variation over time such as muscle/body ratio, and the proportion 
of polar lipids in the muscle. Since the model is based on analytical data; its validity is under the influence of analytical accuracy, and variability between individual fish. It also implies that the sampled fish truly represent the studied populations, quite the case under aquaculture conditions. The model should represent a minimal change of fatty acids: if experimental values are close to the model, the dilution hypothesis can be retained, while changes faster than expected may suggest fatty acid turnover. However a change rate lower than expected seems more difficult to understand. Then results found in neutral lipid of brown trout of LO group are theoretically abnormal, as for most of fatty acids, the changes were lower than expected, with significant differences for $18: 3 n-3,20: 1 n-9,18: 1$ and $20: 1 n-11$ compared to calculated values. That could be explained by possible overestimation of fat gain and illustrates the limitations of the calculations.

When fed diets containing plant oil rich in linoleic and linolenic acids, fish flesh composition is correlatively affected (Thomassen and Røsjø, 1989; Bell et al., 1991; Kalogeropoulos et al.; 1992, Regost, 2001). Then these fatty acids are the most interesting tracers during the washout period before slaughter. However, other minor fatty acids followed the same pattern of changes despite relatively lower initial differences between initial fish fed plant oils and FO fed fish. Compared to these general tendencies, DHA (22:6n-3) is of particular interest. In turbot muscle neutral lipids, the rate of change of this fatty acid was surprisingly lower than expected contrary to other fatty acids. DHA is the major PUFA of fish membranes (Sargent et al., 1999) and is preferentially incorporated in turbot (Linares and Henderson, 1991). Some authors (Tinsley et al., 1973) have found a negative influence of increasing size or age on fish DHA content. In various cases, the percentage of DHA decreases in observed values during the washout despite feeding a FO diet, this may be due to a size effect. A beginning of gonad maturation might also be involved in this low incorporation of DHA in the muscle due to preferential incorporation into gonads (Jeong et 
al., 2002). This relative lower incorporation of DHA in turbot was not seen in brown trout. Although turbot has lower ability to convert PUFA by desaturation and elongation process than salmonids (Cowey et al., 1976; Watanabe, 1982), other influences on intermediate fatty acids in the transformation pathway were not observed. The influence of desaturation ability was mainly observed during feeding fish with vegetal oils (Regost, 2001), as a result polar lipids of trout fed SO diets have higher arachidonic content than control FO group at the beginning of washout phase. After which, fish oil diet did not let to observe any sign of desaturation activities, which could be repressed when their final products were provided into the diet (Ruyter and Thomassen, 1999). The use of control fish as reference to compute model values should also include the effect of specific abilities in FA incorporation and transformation.

Estimation of distances confirm a rather good similarity between fatty acid profiles of computed and experimental values in neutral lipids. It then seems that in neutral lipids, the evolution of fatty acid spectra was relatively close to a dilution for both species. Observed values were closer to the FO reference group than the dilution model, with the exception of neutral lipids of the LO-fed brown trout. Here there was a main tendency to a modification of fatty acids incorporated into neutral lipids only a little faster than the dilution effect. Comparison of this model to other experimental data will be useful to confirm the trends observed here. In the neutral lipids, stored fatty acids seem to be hardly mobilised, mainly when fish are fed to satiation, as here. In rainbow trout, Casteldine and Buckley (1980) suggested there was no turnover of the neutral lipid pool during conditions of positive energy balance.

Muscle phospholipids undergo much faster changes than those predicted by the model. It may be interpreted as reflecting a more active turnover than in neutral lipids, related to the biological activity of phospholipids in cell membranes (Sargent, 1976; Parker et al. 1980). 
External factor such as temperature may also influence incorporation of fatty acids (Tocher and Sargent, 1990) or turn over (Hazel and Neas, 1982). In the experiment used here Turbot were always grown in constant temperature $\left(17^{\circ} \mathrm{C}\right)$, while brown trout were in natural temperature (increasing from 8 to $12^{\circ} \mathrm{C}$ ). It seems logical to evaluate rates of changes in phospholipids considering the increase of total phospholipids in the tissue. However, in the examples studied, the $Q(t) / Q o$ used for phospholipids were lower than that of neutral lipids as lipid content of muscle increased during the course of washout. Then the faster changes in PL were true only compared to the model, but not when absolute changes were compared between NL and PL with the help of relative distances.

The equation (i) of the dilution model can be transformed as :

$$
(\operatorname{Pi}(t)-P i f) /(\text { Pio-Pif })=1 /(Q(t) / Q o)
$$

This equation follows a $y=1 / x$ curve. So, this dilution model provides rather conservative estimates as it predicts an asymptotical tendency to reach a stabilised composition for infinite value of $Q(t) / Q o$ (Figure 1). Variability of experimental data will induce insignificant differences more quickly. However, a longer time (or a higher fat deposition) would be required for a fatty acid initially far away to reach an insignificant difference with its expected final value (as linoleic acid in SO treatment). Because in the examples used here, the time course of changes tended to follow this model, it is assumed that an efficient washout of fish initially fed vegetable oil could take a long time. The evolution of fatty acids in PL looks faster than in NL, only when compared to expected dilution. In any way, relative impact of PL on total lipids is limited in muscle, in the fish species studied here where neutral lipids predominate. 
Figure 1 : Theoretical evolution of fatty acids following the dilution model (curve) plotted as relative decrease of initial differences, between groups previously fed vegetal oils and control fish oil groups, versus relative increase of total fatty acid quantities during growth on the same fish oil diet. Points added correspond to observed values of fatty acids in neutral lipids of muscle from turbot (open symbols) and brown trout (solid symbols) with example of 18:2n-6 in fish previously fed on soybean oil (circles) and 18:3n-3 in fish previously fed on linseed oil (squares) (means \pm standard error).

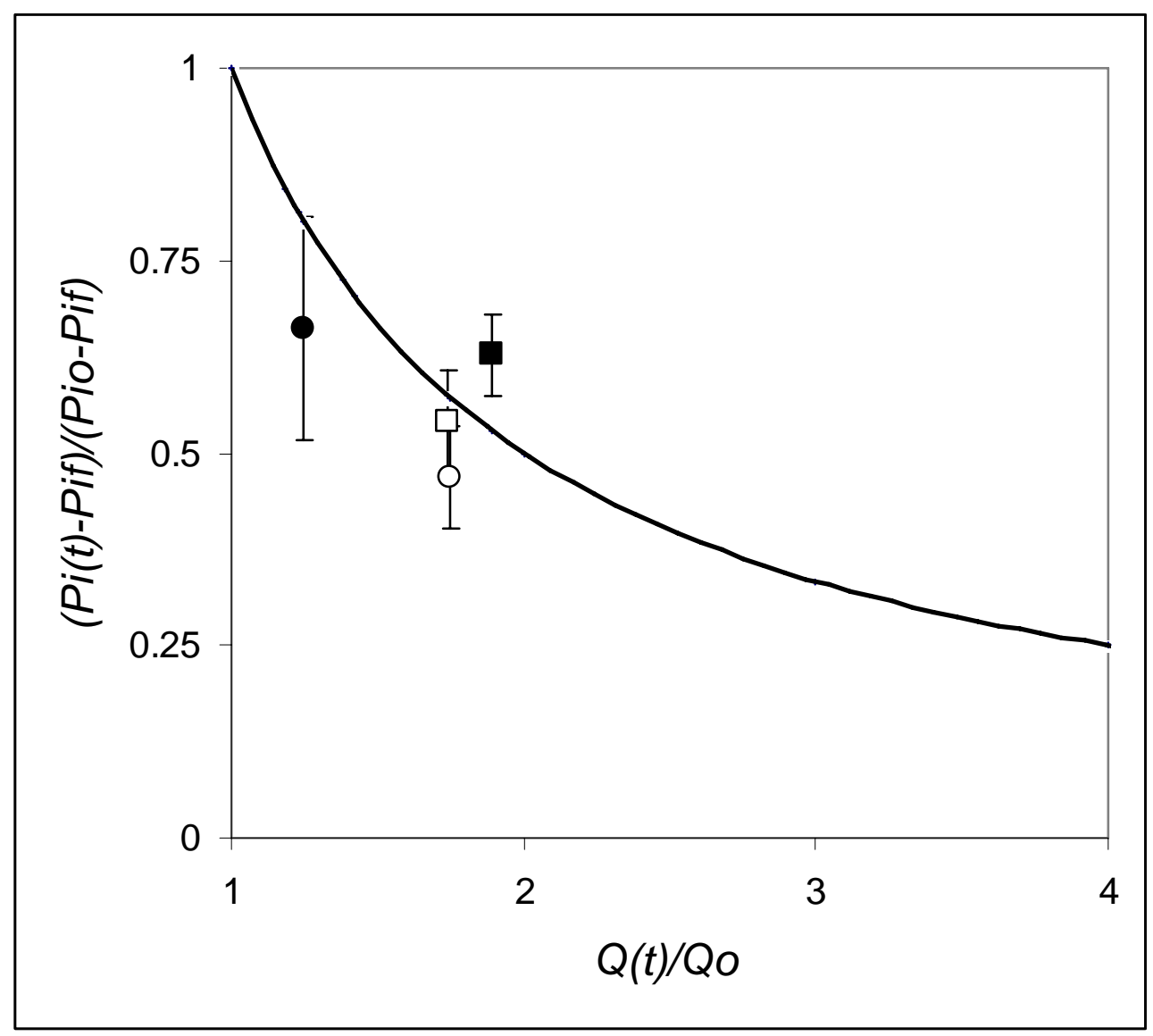

\section{Conclusion}

The dilution of a component into another one is mathematically easy to predict, while in vivo biological processes are more complex. However in order to study regulations, it is of some interest to compare experimental values using simple models. The main interest of this model is to give a reference logical value to estimate how the FA composition evolves after a dietary change. In the studies taken as example for this model, the evolution of neutral lipids followed relatively well this model and so seemed mainly governed by simple dilution. 


\section{References}

Bell, J.G., McVicar A.H,., Park, M.T., Sargent, J.R., 1991. High dietary linoleic acid affects the fatty acid composition of individual phospholipids from tissues of Atlantic salmon (Salmo salar): association with stress susceptibility and cardiac lesion. Lipids 121, $1163-1172$.

Bottino, N.R., 1974. The fatty acids of Antarctic phytoplankton and euphausaiids. Fatty acid exchange among trophic levels of the Ross Sea. Mar. Biol. 27, 197-204.

Castledine, A.J., Buckley, J.T., 1980. Mobility of w3 fatty acids in rainbow trout fed varying levels and types of dietary lipids. J. Nutr. 110, 675-685.

Cowey, C.B., Owen, J.M., Adron, J.W., Middleton, C., 1976. Studies on the nutrition on marine flatfish. The effect of dietary fatty acids on the growth and fatty acid composition of turbot (Scophtalmus maximus). Br. J. Nutr. 36, 479-486.

Dagnelie, P., 1969. Théorie et méthodes statistiques. Duculot, J. Ed, Gembloux France, Vol 2, $463 \mathrm{pp}$.

Folch, J., Lees, M., Sloane-Stanley, G.H., 1957. A simple method for the isolation and purification of total lipids from animal tissues. J. Biol. Chem. 226, 497-509.

Hazel, J.R., Neas, N.P., 1982. Turnover of the fatty acyl and glycerol moieties of microsomal membranelipids from liver, gill and muscle tissue of thermally acclimated rainbow trout, Salmo gairdneri. J. Comp. Physiol. 149B, 11-18

Hardy, R.W., Scott, T.M., Harrell, L.W., 1987. Replacement of herring oil with menhaden oil, soybean oil or tallow in the diet of Atlantic salmon raised in marine net pens. Aquaculture 65, 267-277.

Henderson, R.J., Sargent, J.R., 1985. Chain-length specificities of mitochondrial and peroxisomal beta-oxidation of fatty acids in livers of rainbow trout (Salmo gairdneri). Comp. Biochem. Physiol. 82B, 79-85. 
Jeong, B-Y, Jeong, W-G, Moon, S-K, Ohshima, T., 2002. Preferential accumulation of fatty acids in the testis and ovary of cultured and wild sweet smelt Plecoglossus altivelis. Comp. Biochem. Physiol. 131B, 251-259.

Juaneda, P., Rocquelin, G., 1985. Rapid and convenient separation of phospholipids and nonphosphorus lipids from rat heart using silica cartridges. Lipids 20, 4041.Kalogeropoulos, N., Alexis, M.N., Henderson, R.J., 1992. Effects of dietary soybean and cod liver oil levels on growth and body composition of gilthead bream. Aquaculture $104,293-308$

Kiessling, K.-H., Kiessling, A., 1993. Selective utilization of fatty acids in rainbow trout (Oncorhynchus mykiss Walbaum) red muscle mitochondria. Can. J. Zool. 71, 248-251.

Kiessling, A., Pickova, J., Johansson, L., Asgard, T., Storebakken, T., Kiessling, K-H., 2001. Changes in fatty acid composition in muscle and adipose tissue of farmed rainbow trout (Oncorhynchus mykiss) in relation to ration and age. Food Chem. 73, 271-284.

Linares, F., Henderson, R.J., 1991. Incorporation of ${ }^{14} \mathrm{C}$-labelled polyunsaturated fatty acids by juvenile turbot, Scophthalmus maximus (L.) in vivo. J. Fish Biol. 38, 335-347.

McIntire, C.D., Tinsley, I.J., Lowry, R.R., 1969. Fatty acids in lotic periphyton: another measure of community structure. J. Phycol. 5, 26-32.

Morrison, W.R., and Smith, L.M., 1964. Preparation of fatty acid methyl esters and dimethylacetals from lipids with boron fluoride-methanol. J. Lipid Res. 5, 600-608.

Nettleton, J. A., 1991. w-3 Fatty acids : comparison of plant and seafood sources in human nutrition. J. Am. Diet. Assoc. 91, 331-337.

Parker, R.S, Selivonchick, D.P., Sinnhuber, R. O., 1980. Turnover of label from $1-{ }^{14} \mathrm{C}$ linolenic acid in phospholipids of coho samon, Oncorhynchus kisutch. Lipids 15, 80-85 
Regost, C., 2001. Effets des lipides sur la qualité nutritionnelle, physique et organoleptique de la chair de la truite fario (Salmo trutta) et du turbot (Psetta maxima). Thesis $\mathrm{n}^{\circ} 2498$ University of Rennes I, France.

Regost, C., Arzel, J., Robin, J.H., Rosenlund, G., Kaushik, S.J., 2003. Total replacement of fish oil by soybean or linseed oil with a return to fish oil in turbot (Psetta maxima): growth performance, flesh fatty acid profile and lipid metabolism. Aquaculture 217, $465-482$.

Ruyter, B., Thomassen, S., 1999. Metabolism of n-3 and n-6 fatty acids in Atlantic salmon liver: stimulation by essential fatty acid deficiency. Lipids 34, 1167-1176.

Sargent, J.R., 1976. The structure metabolism and function of lipids in marine organisms. Biochem. Biophys. Perspect. Mar. Biol. 3, 149-212.

Sargent, J., Bell, G., McEvoy, L., Tocher, D., Estevez, A., 1999. Recent developments in the essential fatty acid nutrition of fish. Aquaculture 177, 191-199.

Sérot, T., Regost, C., Prost, C., Robin, J.H., Arzel, J., 2001. Effect of dietary lipid source on odour-active compounds in muscle of turbot (Psetta maxima). J. Sci. Food Agric. 81, $1339-1346$.

Skonberg, D.I., Rasco, B.A., Dong, F.M., 1994. Fatty acid composition of salmonid muscle changes in response to high oleic acid diet. J. Nutr. 124, 1628-1638.

Tidwell, J. H., Robinette, H.R., 1990. Changes in proximate and fatty acid composition of fillet from catfish during a two-year growth period. Trans. Am. Fish. Soc. 119, 31-40.

Tinsley, I.J., Krueger, H.M., Saddler, J.B., 1973. Fatty acid content of coho salmon, Oncorhynchus kisutch - a statistical approach to changes produced by diet. J. Fish. Res. Board Can. 30, 1661-1666 
Tocher, D.R., Sargent, J.R., 1990. Effect of temperature on the incorporation into phospholipid classes and metabolism via desaturation and elongation of n-3 and n-6 polyunsaturated fatty acids in fish cells in culture. Lipids $25,435-442$

Thomassen, M.S., Røsjø, C., 1989. Different fats for salmon: influence on sensory parameters growth rate and fatty acids in muscle and heart. Aquaculture 79, 129-137

Watanabe, T., 1982. Lipid nutrition in fish. Comp. Biochem. Physiol. 73B, 3-15. 